\title{
Deformation and stability of compressible rubber O-rings
}

Brice N. Cassenti ${ }^{1}$ and Alexander Staroselsky ${ }^{2 *}$

\begin{abstract}
Background: In rubber elastic models it is generally assumed that the bulk modulus is infinite, resulting in a material that does not change its volume and a pressure that cannot be evaluated from the material model.

Methods: We have developed a general procedure that incorporates a finite bulk modulus. Using the developed framework accurate results can be obtained without the need for special finite elements. It gives the correct results even in the limit of infinite bulk modulus.

Results: It was shown that material compressibility causes additional stresses mostly associated with an additional hydrostatic pressure. It was also demonstrated that once a bulk modulus is included in the constitutive model, stability analyses of rubber-like materials subject to large deformation become numerically stable and accurate. Hence, it is essential to use compressibility with neo-Hookean solids for accurate stress and lifing predictions. The role of twist in the formation of stress-strain states in rubber O-rings has been evaluated. Such a twist causes elastic instabilities resulting in highly deformed O-ring shapes. Numerical analysis using compressible material models predicted the stable deformed states that O-ring do not remain circular. The ring is buckled and reaches a "chair" type non-planar shape just beyond inside-out twist.

Conclusions: The results indicate that elastic material volume change causes additional stresses mostly associated with an additional hydrostatic pressure. Simulations using various rubber elastic models showed that allowing volume changes allows accurate stress state prediction, reduces numerical difficulties and improves the numerical stability.
\end{abstract}

Keywords: Rubber compressibility, Stability, Large deformation, O-ring

\section{Background}

Rubber-like materials used in the engineering practice often undergo large deformations. Due to superior elastic properties, rubber remains elastic up to very large strain and therefore can be considered as a classical example of finite strain elasticity. A comprehensive survey of variational principles, which form the basis for computational methods and specifically finite element analyses used in this paper, has been completed by Reed and Atluri (1983), Atluri and Cazzani (1995) which also includes mathematical formalism needed to account for large deformation rotations. The strains are well represented by a deviatoric deformation, and show little, or no, volume deformation. Hence most elastic material models neglect volumetric deformation

\footnotetext{
* Correspondence: starosav@utrc.utc.com

2United Technologies Research Center, Department of Physical Sciences, 411

Silver Lane, MS129-73, East Hartford, CT 06108, USA

Full list of author information is available at the end of the article
}

(e.g., see Treloar 1975 or Simo and Taylor 1991) by assuming that the bulk modulus is infinite. Both theoretical and experimental results (Arruda and Boyce 1993; Ali et al. 2010; Ogden 1972, and others) demonstrate that the bulk modulus can be several orders of magnitude larger than the shear modulus justifying the assumption that shear strains will be much larger than the relative volume change. In such cases it can be assumed that the deformations under loading will be governed by the shear response alone. In other words, elastomeric characterization for these materials may be simplified and defined as the ideal incompressibility with infinite bulk modulus, $K$. In reality, rubber materials are not completely incompressible under large strain (Bonet and Wood 1997; Gurvich and Fleischman 2003). Incorporation of relatively small compressibility in the material models reflects physical reality and also allows significant improvements in the finite element method numerical 
predictive procedure. Some reliable and accurate approaches to the experimental evaluation of rubber parameters, finite compressibility, and corresponding results are presented in Gurvich and Fleischman (2003), Gent (2005), Kamiński and Lauke (2013).

The assumption of no volume change leaves the pressure (i.e., the hydrostatic stress) as an unknown that must be found without access to a material response model. Generally, the pressure is found by including it as an unknown at the nodes of a finite element code, which forces the use of special elements with an additional degree of freedom. Of course, with the additional unknown, an additional equation is required to address the fact that there is no volume change. Hence, typical rubber elastic models in finite element codes use special elements for rubber-like materials where the shear modulus is much less than the bulk modulus. Some examples of the finite elements technique with attention to the stability analysis are given in Sussman and Bathe (1987), Duffet and Reddy (1986), and Reese and Wriggers (1995).

In order to eliminate these numerical difficulties, it would be desirable to represent rubber elasticity with a model that can approach the limit of infinitesimal shear modulus with respect to bulk modulus, because typical codes already use shear $G$ and bulk $K$ moduli. Such a formulation would remain accurate for very large bulk-to-shear modulus ratios. This has been the trend recently adopted in MARC (2005), ANSYS, Inc (2004), ADINA (2008). In Ali et al. (2010) a number of compressible material models is presented. Yet most finite element codes contain special zero volumetric strain elements for simulating the response of polymer structures. These models can be combined with other features including structural stability or buckling. Several methods have been suggested to overcome, so-called finite element locking problems (Dolbow and Belytschko 1999) and other numerical instability issues (Reese and Wriggers 1995; Pantuso and Bathe 1997; Gent 2005), which makes it difficult to distinguish physical instabilities from numerical ones. The instabilities place a mathematical constraint that results in nearly singular equations even for structures under conditions far from any buckling load.

In this paper, we propose a method that allows modeling rubber-like materials to approach volume preserving constitutive models gradually. This greatly simplifies the analysis of polymer components. We then apply the technique to a curious instability of polymer O-rings under combined stretch and twist deformation where the possibility of multiple stable states exists.

Most constitutive models are developed either from specific molecular models or from empirical evidence (e.g., see Treloar 1975; Simo and Taylor 1991; Arruda and Boyce 1993; Ogden 1972; Ali et al. 2010; Zéhil and Gavin 2013; Ghaemi et al. 2006; Tabiei and Khambati 2015). We have developed a general procedure that includes small volume changes and, therefore, reduces in the limit to the case of an infinite bulk modulus. We compare the results obtained using compressible and incompressible models and evaluate the predicted stress state and stability sensitivity to the material compressibility. Our general approach allows models generalization to include volumetric deformations in a straightforward manner. In this paper, we use the derivatives of the energy function since the principle stresses $s_{i}$ can be expressed in terms of the energy, $W$, and deviatoric stretch ratios, $\lambda_{i}$, (see, for example, Chadwick and Ogden 1971; Reese and Wriggers 1995). Note that for incompressible materials such a derivative is defined with the unknown additive term for hydrostatic pressure.

A very important engineering problem is estimating the deformation and stresses in polymer O-rings. O-rings are sealing elements that can be used in demanding seal applications over a broad pressure and temperature range. They are easy to assemble, and readily available but require some attention to prevent application problems. See, for example (Ritcher 2016), for a list of specific O-rings failure traces with the damage-causing mechanisms. Polymer O-rings are widely used as seal elements in engineering practice in general and in airplane engines in particular and their deformation is very important, especially due to the fact that seal breakage could lead to the severe oil leakage. As an example from the aerospace industry, failure of the seals could result in engine shutdown and flights that must continue on one remaining engine. During installation, O-ring seals are usually stretched and twisted. Twist can result in an elastic instability for which the shape of the deformed O-ring has more than one equilibrium state. If the O-ring is set on a rigid cylinder, its shape is defined and additional stresses, sometimes very localized, arise. Any instability can place the O-ring in a higher energy state than the original zero deformation reference state that is nevertheless stable. The deformed state of this elastic material causes residual stresses well above what would be predicted for a lower energy state which can lead to cracking of the O-ring. Of course, additional thermal stresses may appear due to heating. This is especially important because the thermal expansion coefficient for rubber-like materials is negative, and the heating of the installed ring will cause additional non-proportional stretching. Hence, for example, in gas turbine engines improper installation can lead to premature seal failure followed by an emergency engine shut down.

Our objective is to develop a mathematical procedure that can be used to naturally extend volume preserving constitutive models to include volumetric strains and eliminate the need for special computational code for rubber-like materials. It was demonstrated that once a bulk modulus is included, analyses for predicting the stability of rubber-like materials subject to large deformation become transparent, since the volumetric deformation is now naturally included. In this paper, it is shown that small variations 
in the compressibility (bulk modulus) might lead to the significant changes in predicted stress-strain state, which is close to the results of Gent et al. (2007) and Destrade et al. (2012). Therefore, the use of compressibility with neoHookean solids is essential.

We begin our discussion with a detailed presentation of a method for extending volume preserving models to models with volumetric deformations. The extension naturally includes the original volume preserving model as a limiting case.

The following section summarizes a generic method for including a bulk modulus in rubber elastic materials. For the sake of clarity, we use the Mooney-Rivlin constitutive model in this section to illustrate the approach. In Section 3, we then include several examples illustrating the reduction of the model to commonly used engineering problems. In Section 4, the deformation and the multiple stable states that can exist in polymer O-rings are discussed. This section includes a discussion on the elastic stability of O-rings. We demonstrate that including volumetric deformations in the material model significantly increases the accuracy of the predictions. We finish with some concluding remarks.

\section{Methods}

\section{Governing equations}

Rubber-like materials possess relatively low shear moduli while supporting extremely large elastic strains. This makes standard small strain elasticity inappropriate for analyzing real components such as O-rings. Even for small strain cases, the equations of linear elasticity need to be modified since the bulk moduli are much larger than the shear moduli. Note that indicial notation will be used throughout where repeated indices are assumed to be summed over the number of dimensions, and commas indicate partial derivatives (or more generally covariant derivatives). Using indicial notation, the equilibrium equations for incompressible materials can be summarized, from Treloar (1975), as:

$$
s_{j i, j}+P_{, i}=0,
$$

where $s_{j i}$ is the deviatoric stress components, and $P$ will later be shown to be related to the hydrostatic pressure. The stress is decomposed into its hydrostatic and deviatoric stress to allow analyses for an infinite bulk modulus case. The quantity $P$ is required since the material is assumed to be incompressible (i.e., $u_{i, i}=0$, where $u_{i}$ is the displacement vector). Assuming incompressibility adds a constraint, but this removes any reference to the hydrostatic pressure making it an additional unknown that must satisfy the equilibrium equations. One may see that the system (1) can be generally resolved within an additive parameter $P$ as in the description of viscous flow. In other words, when compared to classical elasticity the pressure is now an independent variable, and there is a constraint on the displacement gradient. Many finite element codes use special elements for simulations using the above equations. Such a model could be numerically unstable but in most practical cases the accuracy should be sufficient, if higher precision is used.

\section{Modification of classical rubber elasticity}

In this section, we will modify classical rubber elasticity to include volume changes in a straight forward manner that will remain valid for large strains. Since the shear strains are large, kinematics is an important first step in the development of a generic framework. The formulation in Eq. (1) needs to be extended. We will remove the requirement that $\boldsymbol{u}_{i, i}=0$ by adding a change in volume to the governing equations in a manner that still allows the user to proceed to the limiting case of a volume preserving material. We begin by decomposing the deformation gradient, $\boldsymbol{F}$ into a rotation, $\boldsymbol{R}$, and a symmetric right stretch tensor, $\boldsymbol{U}$. The stretch tensor can be decomposed into the product of a dilation, $\underline{\Delta}$, and a deviatoric stretch, $\underline{\Lambda}$, or

$$
F_{\mathrm{ij}}=R_{i k} U_{k j}=R_{\mathrm{i} k} \Delta_{k l} \Lambda_{l j} .
$$

The dilation is a diagonal tensor given by

$$
\Delta_{i j}=J^{1 / 3} \delta_{\mathrm{ij}}
$$

where

$$
J=\operatorname{det}\{\mathbf{F}\}
$$

and, hence, $\operatorname{det}\{\underline{\Lambda}\}=\operatorname{det}\{\boldsymbol{R}\} \equiv 1$.

Kinematics and equilibrium are not sufficient to calculate the stress in the structure; we also need a mechanically motivated constitutive relationship for non-linear elasticity.

We will follow an approach described in Cassenti and Staroselsky (2000) and Ghaemi et al. (2006). For simplicity, without loss in generality, Mooney-like models will be modified to include a finite bulk modulus. This modification can be used to illustrate the procedure for including volume changes that can approach, in the limit, the case of classical rubber elasticity. For an isotropic compressible $(J \neq 1)$ rubber-like material, the strain energy $(W)$ can be written in terms of the first invariant of the principal deviatoric strain ratios $\left(\lambda_{1}, \lambda_{2}, \lambda_{3}\right)$ and the volume ratio $(\eta)$ as

$$
W=\frac{1}{2} G\left(\lambda_{1}^{2}+\lambda_{2}^{2}+\lambda_{3}^{2}-3\right)+\frac{1}{2} K(J-1)^{2}
$$

Note that the principal deviatoric stretch ratios are $\left(\lambda_{1}, \lambda_{2}, \lambda_{3}\right)$ are the eigenvalues of $\Lambda$ and $J$ is the final-toinitial volume ratio. The parameters $G$ and $K$ are the elastic moduli, which will be shown later to be the shear and bulk moduli, respectively. Let the principal stretch ratios, $\mu_{i}$ be the eigenvalues of the right stretch tensor $\boldsymbol{U}$ then from Eq. (2). 


$$
\mu_{i}=J^{1 / 3} \lambda_{i} i=1,2,3
$$

Recall that, since the deviatoric part of the deformation is always volume preserving, the deviatoric stretch ratios satisfy

$$
\lambda_{1} \lambda_{2} \lambda_{3}=1
$$

and the right-hand stretch ratios $\left(\mu_{i}\right)$ include the volume change (i.e., $\mu_{1} \mu_{2} \mu_{3}=\jmath$ ). After the rotation is removed, the Biot stresses $\left(s_{\mathrm{ij}}\right)$, which are energy conjugate to the right-hand stretch tensor, and, hence, the principal stresses $\left(s_{1}, s_{2}, s_{3}\right)$ are conjugate to the principal stretch ratios $\left(\mu_{1}, \mu_{2}, \mu_{3}\right)$ therefore the incremental work done by the stresses has the following form:

$$
d W=s_{1} d\left(J^{1 / 3} \lambda_{1}\right)+s_{2} d\left(J^{1 / 3} \lambda_{2}\right)+s_{3} d\left(J^{1 / 3} \lambda_{3}\right)
$$

By differentiation, the constraint (7) can be rewritten as

$$
\frac{d \lambda_{1}}{\lambda_{1}}+\frac{d \lambda_{2}}{\lambda_{2}}+\frac{d \lambda_{3}}{\lambda_{3}}=0
$$

The constraint (9) is difficult to incorporate directly in the constitutive equations, but if a cyclic change of variables is introduced as follows:

$$
\begin{aligned}
& d \lambda_{1}=\lambda_{1}\left(d \alpha_{2}-d \alpha_{3}\right), \\
& d \lambda_{2}=\lambda_{2}\left(d \alpha_{3}-d \alpha_{2}\right), \\
& d \lambda_{3}=\lambda_{3}\left(d \alpha_{1}-d \alpha_{2}\right)
\end{aligned}
$$

where the new variable $\alpha_{i}$, can be used to replace the deviatoric stretch ratio, the derivation is much clearer. Equation (10) is the key to developing constitutive relations that can gradually approach volume preserving representations. Note that cyclic permutations show that only the differences in the differentials of $\alpha_{i}$ are required in Eq. (10).

First, we expand the differentials in Eq. (8). Next, we substitute for the deviatoric stretch ratios using Eq. (10) to obtain:

$$
\begin{aligned}
d W=J^{1 / 3} & {\left[s_{1} \lambda_{1}\left(d \alpha_{2}-d \alpha_{3}\right)+s_{2} \lambda_{2}\left(d \alpha_{3}-d \alpha_{1}\right)\right.} \\
& \left.+s_{3} \lambda_{3}\left(d \alpha_{1}-d \alpha_{2}\right)\right] \\
& +\left(s_{1} \lambda_{1}+s_{2} \lambda_{2}+s_{3} \lambda_{3}\right) \frac{d J}{3 J^{2 / 3}}
\end{aligned}
$$

The constitutive equation for the strain energy, (5), can be used to find the change in the internal energy stored in the material in the following way:

$$
d W=G\left(\lambda_{1} d \lambda_{1}+\lambda_{2} d \lambda_{2}+\lambda_{3} d \lambda_{3}\right)+K(J-1) d J
$$

and must be equal to the work done by the stresses as shown in Eq. (8). Substituting for the stretch ratios in Eq. (12) and using expressions (10) we immediately obtain $d W=G\left[\lambda_{1}^{2}\left(d \alpha_{2}-d \alpha_{3}\right)+\lambda_{2}^{2}\left(d \alpha_{3}-d \alpha_{1}\right)+\lambda_{3}^{2}\left(d \alpha_{1}-d \alpha_{2}\right)\right]+K(J-1) d J$

Since the quantities $d \alpha_{i}$ and $d J$ are arbitrary, they must apply in all possible deformation states. Since Eqs. (11) and (13) are written for the same energy change, they imply

$$
J^{1 / 3}\left(s_{i} \lambda_{i}-s_{j} \lambda_{j}\right)=G\left(\lambda_{i}^{2}-\lambda_{j}^{2}\right) ; \quad i \neq j ; \quad i, j=1,2,3
$$

And

$$
\lambda_{1} s_{1}+\lambda_{2} s_{2}+\lambda_{3} s_{3}=3 J^{2 / 3} K(J-1)
$$

Note that there are only two independent Eqs. in (14). Equations (14) are satisfied by

$$
s_{i}=G \frac{\lambda_{i}}{J^{1 / 3}}+\frac{P}{J^{1 / 3} \lambda_{i}}
$$

where the quantity $P$ can be found by substituting Eq. (16) into Eq. (15) to yield

$$
P=K J(J-1)-\frac{1}{3} G\left(\lambda_{1}^{2}+\lambda_{2}^{2}+\lambda_{3}^{2}\right)
$$

For completeness, the principal Biot stresses for all possible deformations, with finite bulk modulus, can now be written as

$$
\begin{gathered}
s_{i}=\frac{G}{J^{1 / 3} \lambda_{i}}\left(\frac{2}{3} \lambda_{i}^{2}-\frac{1}{3} \lambda_{k}^{2}-\frac{1}{3} \lambda_{l}^{2}\right)+\frac{K J^{2 / 3}(J-1)}{\lambda_{i}} . \\
i \neq k \neq l ; i, k, l=1,2,3
\end{gathered}
$$

Note that in Eq. (18), principal stresses are used, and that the Biot is conjugate to the right-hand stretch tensor. Equation 18 indicates that it is not the bulk modulus, $K$, alone or the relative volume change, $J-1$, alone that controls whether a constitutive law is volume preserving, but the product of the bulk modulus and the relative volume change that is important. For $J=1$, and $K$ finite, that is the case of pure deviatoric deformation but with volume changes possible, the sum of the stresses in Eq. (14) indicates that the first term in Eq. (18) is related to the deviatoric stress and, of course, the second term is the hydrostatic component.

\section{Examples}

In this section, we examine the applicability of the derived modification for constitutive laws in some classical examples. Throughout the next section, we assume a Mooneylike model for the sake of simplicity.

Several examples can be used to illustrate the development of constitutive relations for rubber-like materials. The examples will show in a straight forward manner the ease with which the material response can be developed for isotropic materials with a large bulk modulus relative to the 
shear modulus. Three examples will be used: (1) small strain response, (2) hydrostatic response, and (3) uniaxial response.

\section{Small strain response}

For small strains, let the principal deviatoric stretch ratio be given by

$$
\lambda_{1}=1+e_{1}, \quad \lambda_{2}=1+e_{2}, \quad \text { and } \quad \lambda_{3}=1+e_{3},
$$

where $e_{1}, e_{2}, e_{3}<<1$. Equation (7) to first order in $e_{\mathrm{i}}$ yields

$$
e_{1}+e_{2}+e_{3}=0 .
$$

Let the volume ratio be

$$
J=\frac{V_{f}}{V_{i}}=1+\frac{\Delta V}{V_{i}}=1+v,
$$

where $V_{f}$ is the current volume element, $V_{i}$ is the initial volume, $\Delta V=V_{f}-V_{i}$, and $v=\frac{\Delta V}{V_{i}}<<1$. Then to first order in the strains expression (21) yields

$$
s_{i} \approx 2 G e_{i}+K v \quad \text { for } i=1,2,3 \text {. }
$$

Expression (22), together with Eq. (20) is the correct form for small strains with a volume change and demonstrates that $G$ is the shear modulus while $K$ is the bulk modulus.

\section{Hydrostatic response}

Next, let us consider the case of hydrostatic stress. Since the deformation only includes volume change,

$$
\lambda_{1}=\lambda_{2}=\lambda_{3}=1
$$

and set

$$
s_{1} / J^{2 / 3}=s_{2} / J^{2 / 3}=s_{3} / J^{2 / 3}=p .
$$

Then from Eq. (17)

$$
p=K(J-1)
$$

is the hydrostatic tension. Note that

$$
p=s_{1} /\left(\lambda_{2} \lambda_{3} J^{2 / 3}\right)=s_{2} /\left(\lambda_{3} \lambda_{1} J^{2 / 3}\right)=s_{3} /\left(\lambda_{1} \lambda_{2} J^{2 / 3}\right),
$$

demonstrates that $p$ is the hydrostatic stress with respect to the current area.

\section{Uniaxial response}

An important example is that of uniaxial stress. Take the stress to be along direction 1 , then

$$
s_{2}=s_{3}=0 .
$$

The stretch ratios perpendicular to direction 1 are, using Eq. (7),

$$
\lambda_{2}=\lambda_{3}=\frac{1}{\sqrt{\lambda_{1}}} .
$$

From Eq. (18)

$$
J^{1 / 3} \lambda_{2} s_{2}=0=K J(J-1)-\frac{G}{3}\left(\lambda_{1}^{2}-\frac{1}{\lambda_{1}}\right)
$$

Equation (27a) can be solved for $J-1$ to yield

$$
J-1=\frac{G}{3 K J}\left(\lambda_{1}^{2}-\frac{1}{\lambda_{1}}\right)
$$

Substituting Eqs. (26) and (27) into Eq. (18) gives the uniaxial stress as

$$
s_{1}=\frac{G}{J^{1 / 3}}\left(\lambda_{1}-\frac{1}{\lambda_{1}^{2}}\right)
$$

For the case of small volume changes Eq. (28) becomes, using Eq. (27b),

$$
s_{1}=G\left(\lambda_{1}-\frac{1}{\lambda_{1}^{2}}\right)\left[1-\frac{G}{9 K}\left(\lambda_{1}^{2}-\frac{1}{\lambda_{1}}\right)\right] .
$$

Equation (29) agrees with the classical model (Treloar 1975) for incompressible rubber elasticity which corresponds to the limit $G / K \rightarrow 0$.

\section{Results and discussion}

Deformation and stability of rubber O-rings

Polymer O-rings are widely used as seal elements in engineering practice (see the picture in Fig. 1). During installation, these rings are usually stretched and twisted. We evaluate the role of axisymmetric torsion and twist in the formation of stress-strain state in the ring.

This is related to the manufacturing process when the ring may be installed inside-out due to twisting. For example, if an O-ring is twisted about the major circumference so that the outer major circumference is on the

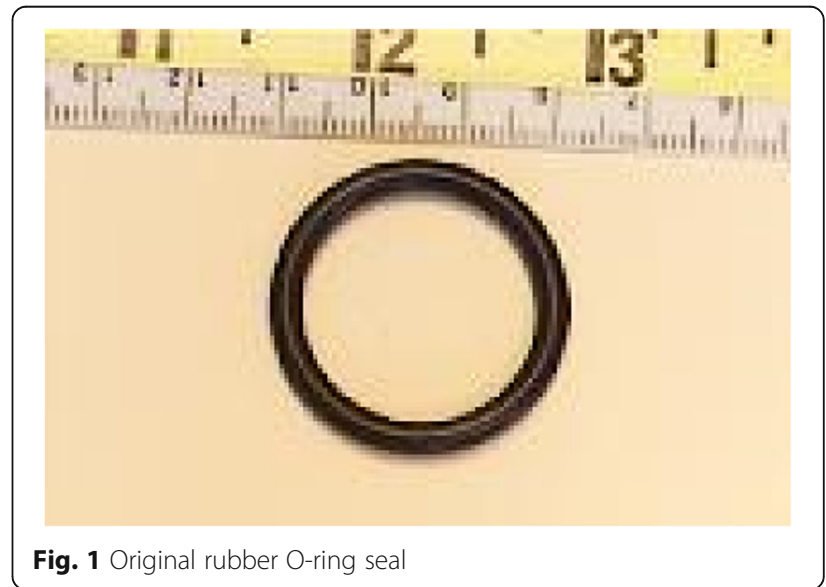


inside, the O-ring will be in a state of unstable neutral equilibrium. Similarly, if a uniform hydrostatic pressure is applied, the volume change will be small, but, because of the small shear modulus, non-uniformity in the loads or the geometry can result in significant O-ring deformations. Both the twisting and pressure instabilities are geometric and are important in predicting the final state. Thus, the shape of the (twisted) deformed ring is not round anymore (so called Michell's instability (Goriely 2006). ${ }^{1}$ If the ring is set on a rigid cylinder, its shape is defined and additional stresses, sometimes very localized, arise that in turn may lead to the ring cracking. We have analyzed two related problems, namely, (i) homogeneous axisymmetric twisting of the ring and (ii) the full three-dimensional twisting-torsion problem for the ring. Under certain physically based assumptions, the first problem allows a closedform solution. The second problem is solved numerically using the finite element technique. For the model calibration, we obtain an analytical solution for the axisymmetric O-ring torsion problem $(i)$ and compare it with the numerical predictions and show that both results are accurate.

First of all, we start with the kinematics (i.e., the motion of points in the material) and consider axisymmetric torsion. We assume that during the deformation each crosssection (circle with the radius $r_{0}$ ) remains in its original plane. We also assume that due to symmetry of the problem, the principal strains are in hoop, radial, and circumferential directions. This greatly simplifies the conversion from Green-Lagrange strain and second Piola-Kirchoff stress to right-hand stretch and Biot (or Jaumann) stress. Axisymmetric torsion as shown in Fig. 2 implies that the primary principal strain and stress appear in the hoop direction. During the rotation through the angle $\Theta$, the curve $A A^{\prime}(l=2 \pi R)$ moves to the curve $B B^{\prime}(l=2 \pi(R$ $+\Delta R))$. One can see that the extension is $\Delta \lambda=2 \pi \Delta R$ or $\lambda_{1}$ $=(R+\Delta R) / R$. The sign and magnitude of this deformation depend on the position of point $A$ (or angle $\alpha$ ) and on the position of point $B$ (or angle $\Theta$ ), as well as on radii $R_{O}$ and $\rho, \rho \leq r_{0}$. It is also important that, usually, the ratio ${ }^{r_{0}} / R_{0}$ is much smaller than unity. Under these assumptions, the stretch ratio $\mu_{1}=J^{1 / 3} \lambda_{1}$ in the hoop direction is given by

$$
\mu_{1}=\frac{1-\frac{\rho}{R_{0}} \cos (\alpha+\theta)}{1-\frac{\rho}{R_{0}} \cos \alpha}+O\left(\left(\frac{r_{0}}{R_{0}}\right)^{2}\right)
$$

By this point, we have not used any material-specific parameters or behavior. In order to calculate the state of the $\mathrm{O}$-ring, we introduce the specific stress-strain relations for the analyzed materials (i.e., we introduce the constitutive model). A good constitutive model should represent the three-dimensional nature of stress-stretch behavior using a minimal number of parameters to represent physically the deformation process. The eight chain model of Arruda and Boyce (1993) accurately captures the cooperative nature of network deformation while requiring only two material parameters, an initial shear modulus $G$ and limiting chain extensibility where the parameter $N$ is the number of chemical cross-links per length. This parameter is related to the locking stretch as follows: $\lambda_{L}=\sqrt{N}$. We modify this model by incorporating the term reflecting the volume changes ${ }^{2}$ where the energy function (i.e., the Hemholtz free energy), $W$ has been taken in the form:

$$
W=G\left(\frac{1}{2}\left(I_{1}-3\right)+\frac{1}{20 N}\left(I_{1}^{2}-9\right)+\frac{11}{1050 N^{2}}\left(I_{1}^{3}-27\right)+\ldots\right)+\frac{1}{2} K(J-1)^{2}
$$

where $I_{1}=\lambda_{1}^{2}+\lambda_{2}^{3}+\lambda_{3}^{2}$ is the first invariant of the deviatoric diagonal stretch tensor and $K$ is a bulk modulus. Next, if we consider, for simplicity, the case $N=\infty$, it would correspond to a one term non-volume preserving material model.

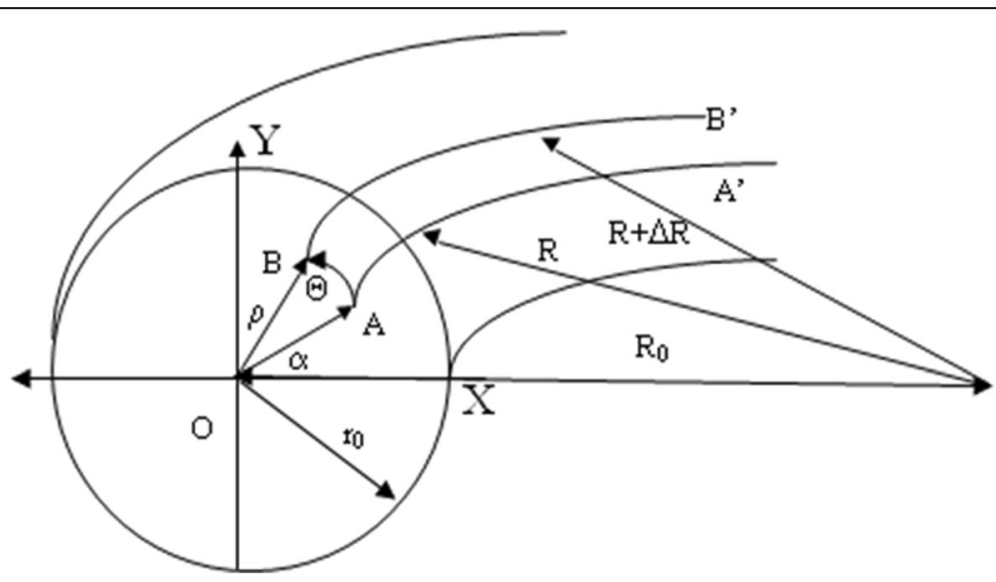

Fig. 2 Cross-section and part of the twisted O-ring 
Material models, such as Eq. (31), allow the analysis of arbitrary stress states, including ones for materials with an infinite bulk modulus. This makes possible a direct evaluation of the effects of a finite bulk modulus. We calibrated an incompressible constitutive model by comparing the model predictions against test results for simple tension up to $200 \%$ deformation. As shown in Fig. 3, finite element uniaxial calculations using parameters with values of $G=160$ psi; $N=$ 18 resulted in curve-fitting errors of less than $5 \%$ for the Oring material if considered. In order to match experimental data, we also adopted the value of ${ }^{G} / K=0.1$ allowing compressibility. The bulk modulus is ten times larger than the shear modulus, and the volume change, $J-1$, is small.

Our analysis of O-rings for axisymmetric torsion, a rotational displacement was specified as shown in Fig. 2. We have considered two model cases: compressible and incompressible. The first model allows for the volume change, which increases in the "tension" zone and decreases under compression. From pure geometrical considerations, it is easy to see that the volume ratio is given by $J=\mu_{1}$. Note that the remaining two principal stretch ratios are unity (i.e., $\mu_{2} \equiv J^{1 / 3} \lambda_{2}=\mu_{3} \equiv J^{1 / 3} \lambda_{3}=1$ ) since there is no strain in the plane of the cross-section. Using Eq. (18) and the fact that the product of the deviatoric stretch ratios is unity $\lambda_{1} \lambda_{2} \lambda_{3} \equiv 1$, we immediately obtain for the hoop Biot stress

$$
\frac{s_{1}}{G}=\frac{2}{3} J^{1 / 3}\left(1-\frac{1}{J^{2}}\right)+\frac{K}{G}(J-1)
$$

The graphs of stress calculations for two surface points separated by $90^{\circ}$ angle versus rotation angle of the O-ring are shown in Figs. 4 and 5. Note that $J$ is calculated based on the hypothesis of cross-sections remaining planar.

The second case considered is based on the additional assumption of incompressibility of rubber-like material. It immediately leads to $\mu_{i}=\lambda_{i}$, for all principal directions or in other words it leads to $J=\lambda_{1} \lambda_{2} \lambda_{3} \equiv 1$. Since we have assumed a uniaxial stress state, the hoop stretch ratio equals $\lambda$ and the remaining two principal stretch ratios can now be found as $1 / \sqrt{\lambda}$. The uniaxial stress is calculated by substituting for the stretch ratios in the expression (31) for the elastic energy (the material constitutive model with $J=1$ ) and differentiating with respect to the stretch as follows:

$$
\frac{s_{1}}{G}=\left(\lambda^{2}-\frac{1}{\lambda}\right)+\frac{1}{5 N}\left(\lambda^{2}+\frac{2}{\lambda}\right)\left(\lambda^{2}-\frac{1}{\lambda}\right)+\ldots
$$

The graphs of stress calculations based on these model assumptions are also presented in Figs. 4 and 5 together with finite element method results. This comparison between numerical and analytical solutions has been used to verify the finite element model predictions. A finite element code was used to perform numerical investigations. Note that the MARC (2005) finite element code developed the governing equations using a virtual displacement formulation based on the Green-Lagrange strain and the second Piola-Kirchoff stress. Hence, equilibrium (i.e., the conservation of momentum) is automatically satisfied. A user-material subroutine was written to incorporate the Aruda-Boyce model and a finite bulk modulus as in Eq. (31). We compared the second Piola-Kirchhoff stress predictions (the conversion from Biot stress to second PiolaKirchoff, and the conversion from Green-Lagrange Strain

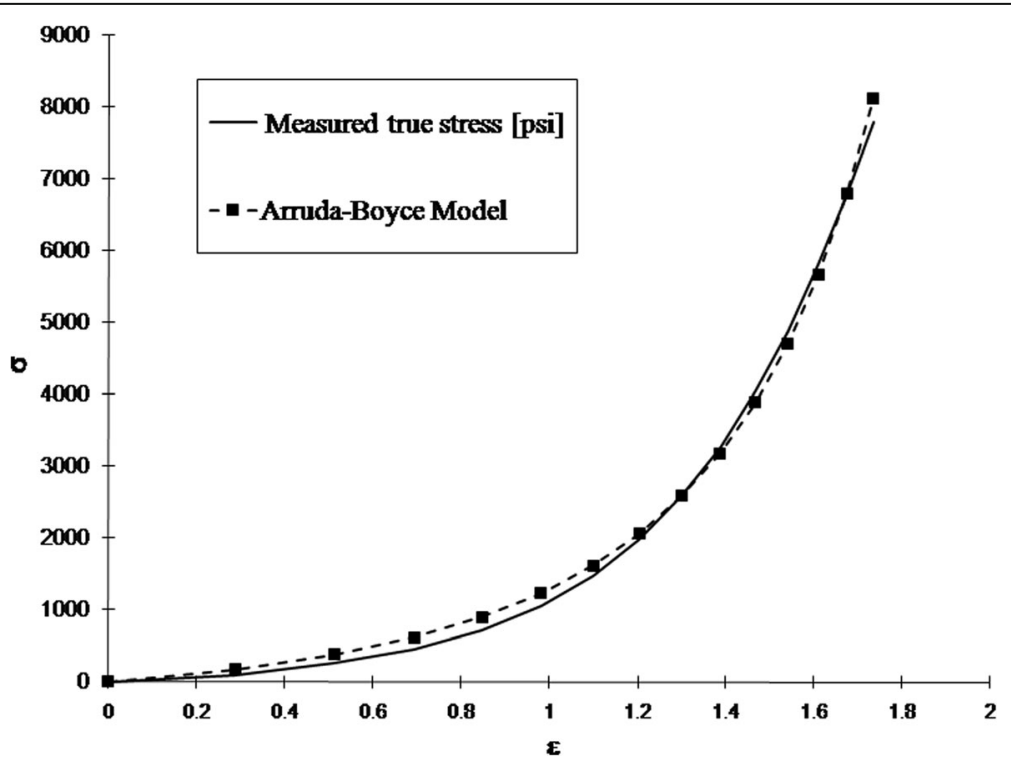

Fig. 3 Experimental and predicted true stress-strain curves for tension. Fitting of the test data has been done by Arruda-Boyce model with $G=160$ psi and $N=18$ 


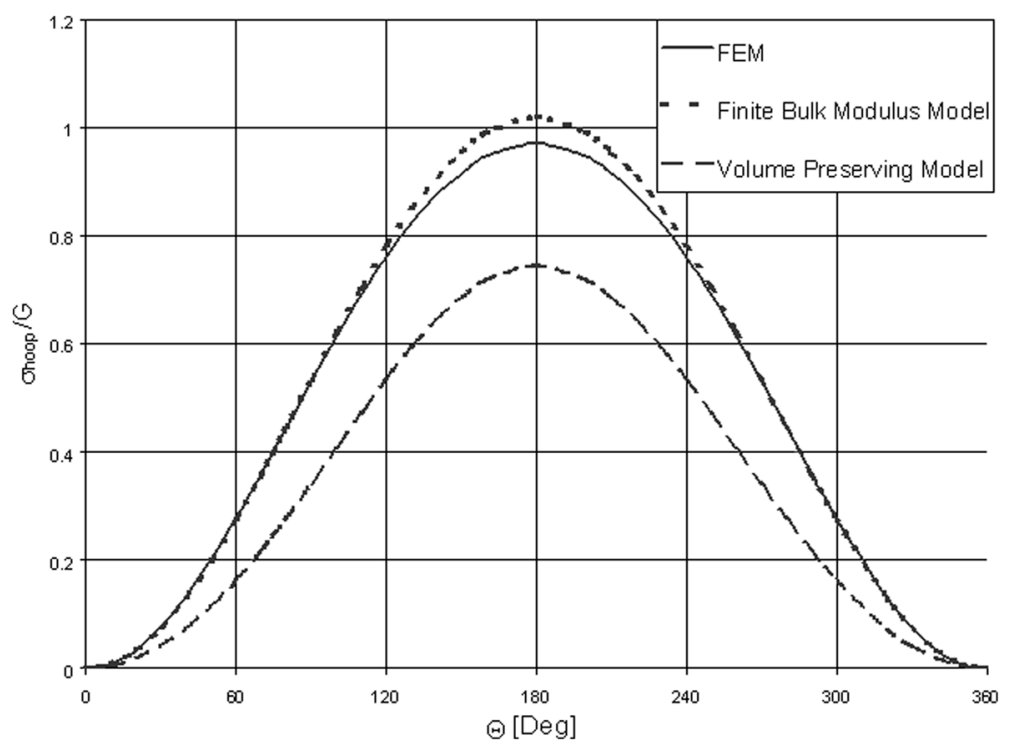

Fig. 4 Normalized true stress vs. angle for inside-out rotation (point $X$ at Figure 2) $a=0$.

to right-hand stretch are given in the Appendix) from the code numerical simulations with the hoop stress $s_{1}$ predictions calculated according to formulae (32) and (33). The comparisons shown in Figs. 4 and 5 have been performed for two initial positions: the inside point $\mathrm{X}(\alpha=0)$ in Fig. 2 and for the point on the top of the ring ( $\mathrm{Y}$ in Fig. 2, $\alpha=$ $90^{\circ}$ ). These results clearly indicate that material volume changes cause additional stresses, mostly associated with an additional hydrostatic pressure. As one may see from these figures, kinematic boundary conditions imposed in the finite element code are much closer to the first type of the axisymmetric problems (Eq. (32)). The results show that "inside-out" twist $\left(\Theta=180^{\circ}\right)$ causes maximum principal stress for the points laying on the horizontal plane (for example point $\mathrm{X}$ in Fig. 2) and is in tension for the points originally close to the O-ring center and compression for

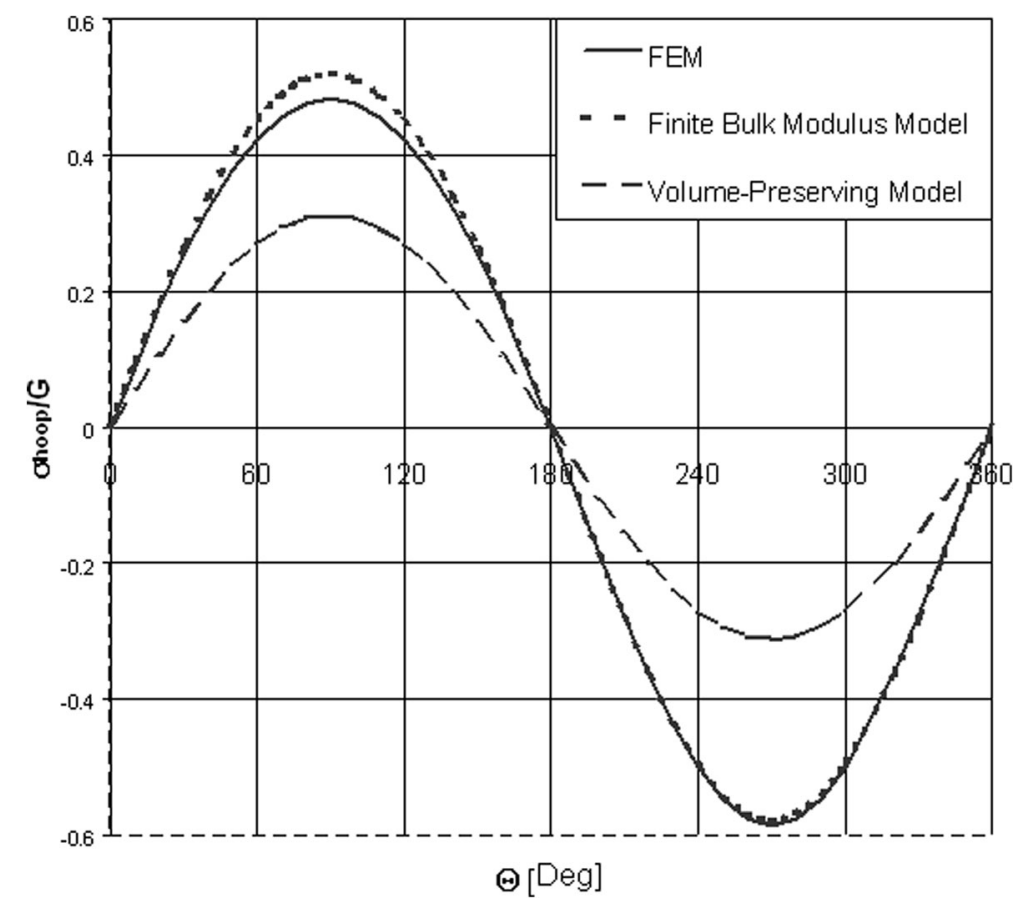

Fig. 5 Normalized true stress vs. angle for rotation of the point $Y: a=\pi / 2$. 
the "outer" region. For points in the cross-section normal to the O-ring plane, the most critical twist is $\Theta=90^{\circ}$ where the maximum stress is about half of the inside-out twist level, which coincides with our intuitive expectation. Finally, we see that half of the O-ring torus is in tension, and the other half is under compression. Of course, this is just a reflection of the state of internal equilibrium.

We developed our simulations using the compressible Aruda-Boyce model and applied it to the finite element analysis of several full 3D loading conditions. The most illustrative and interesting from an experimental point of view case is twisting of one side of the O-ring while the opposite part $\left(180^{\circ}\right.$ apart $)$ is to be held. This generates an extremely inhomogeneous deformation state. One may see that the ring has a new equilibrium state after a $180^{\circ}$ rotation, and this equilibrium shape does not remain round and has a so-called "chair"-type shape. A comparison between FEM predictions and the actual O-ring shape is shown in Fig. 6. It was suggested in Cassenti and Staroselsky (2000) that the O-ring loses stability when it is twisted by about two full turns. Our numerical analysis and corresponding experiments (see Fig. 6), however, demonstrated that even one full rotation already causes the so-called Michell's instability, and the original ring obtains a non-round stable shape. In addition, if the O-ring is held on its inside by a rigid cylinder, extremely localized stresses will arise and could lead to premature failure. The model predicts the deformation extremely well. The maximum principal stress reaches $0.35 \mathrm{G}$ at the fixed inner point. The stress at the "moving" inner O-ring point is significantly smaller as shown in Fig. 7a, b. Note the minor numerical instability around the peak stresses in Fig. $7 \mathrm{~b}$.

Two cases of interest are: (a) O-ring is twisted at opposite ends of the major diameter in opposite directions and (b) O-ring is twisted at opposite ends of the major diameter in the same direction. Figures 8 and 9 present the deformed shapes with contours of the hoop stress along the major axis. When the applied moments are in opposite directions, the O-ring is curved up. If moments act in the same direction (Fig. 9), one end is up and the other down, exhibiting an inflecting point. Also note that the stress contours are discontinuous where the moments are applied. These stable states are readily observed using common O-rings.

During service, especially in the engine applications, Orings are subject to the thermal exposure. Typically rubber thermal expansion coefficients are negative and of the order of $\alpha=-200 \cdot 10^{-6} K^{-1}$. Thus, due to the temperature increase, the rubber O-ring contracts generating additional stretch $\delta=1+\varepsilon_{\text {therm }}=1+\left|\alpha_{\text {therm }}\right| \Delta T$. Therefore, for the particular case of combined stretch and axi-symmetric torsion it could be written:

$$
\mu_{\text {total }}=(1+\delta) \mu_{1} \approx(1+\delta)\left(1+2 \varepsilon \sin \left(\alpha+\frac{\theta}{2}\right) \sin \left(\frac{\theta}{2}\right)\right) ;
$$
where $\delta$ is the stretch and $\varepsilon=\rho / R_{0}$. Note that we modified (30) using a Taylor expansion. For the general full 3D problem, when stretch principal extensions are not parallel to the torsion-twist ones the problem is more complicated and may be analyzed numerically.

Excessive temperature exposure can cause surface cracks on the O-ring and also results in material properties degradation and permanent deformation (Ritcher 2016). However, even performance under temperature variations below acceptable temperature limits leads to crack formation, due to high stresses caused by combined stretch and axisymmetric torsion as shown above.

The critical load that O-ring can sustain depends on the amount of twist and maximum operational temperature. Based on (30) and (32), one can see that the thinner O-ring (the smaller the ratio $r_{0} / R_{0}$ ), the higher the twist deformation needed to reach to generate similar stress level. The engineering analysis

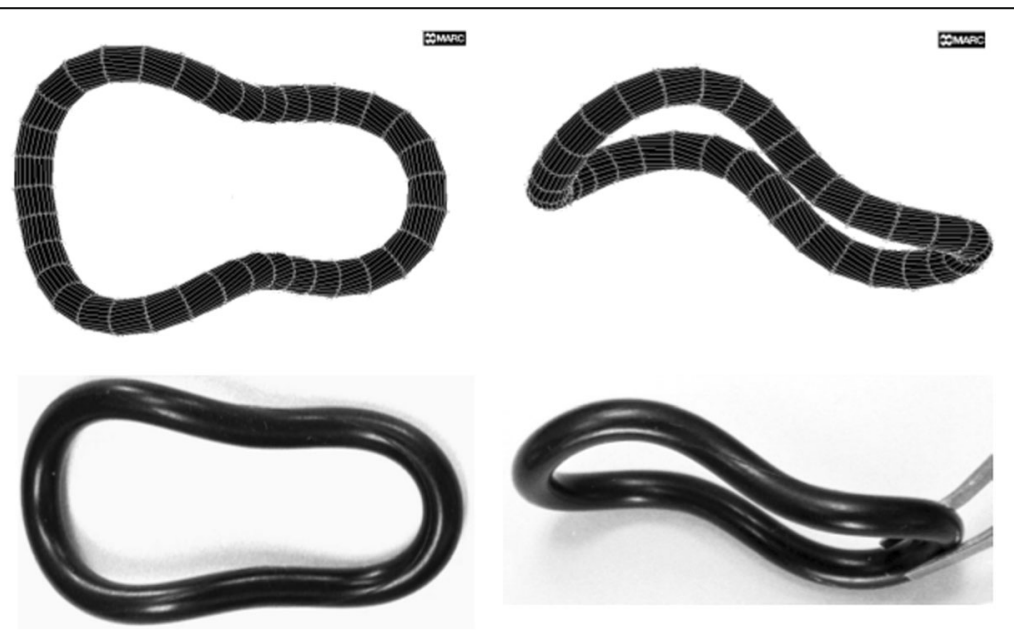

Fig. 6 O-ring twist deformation: FEM predictions vs. nature 

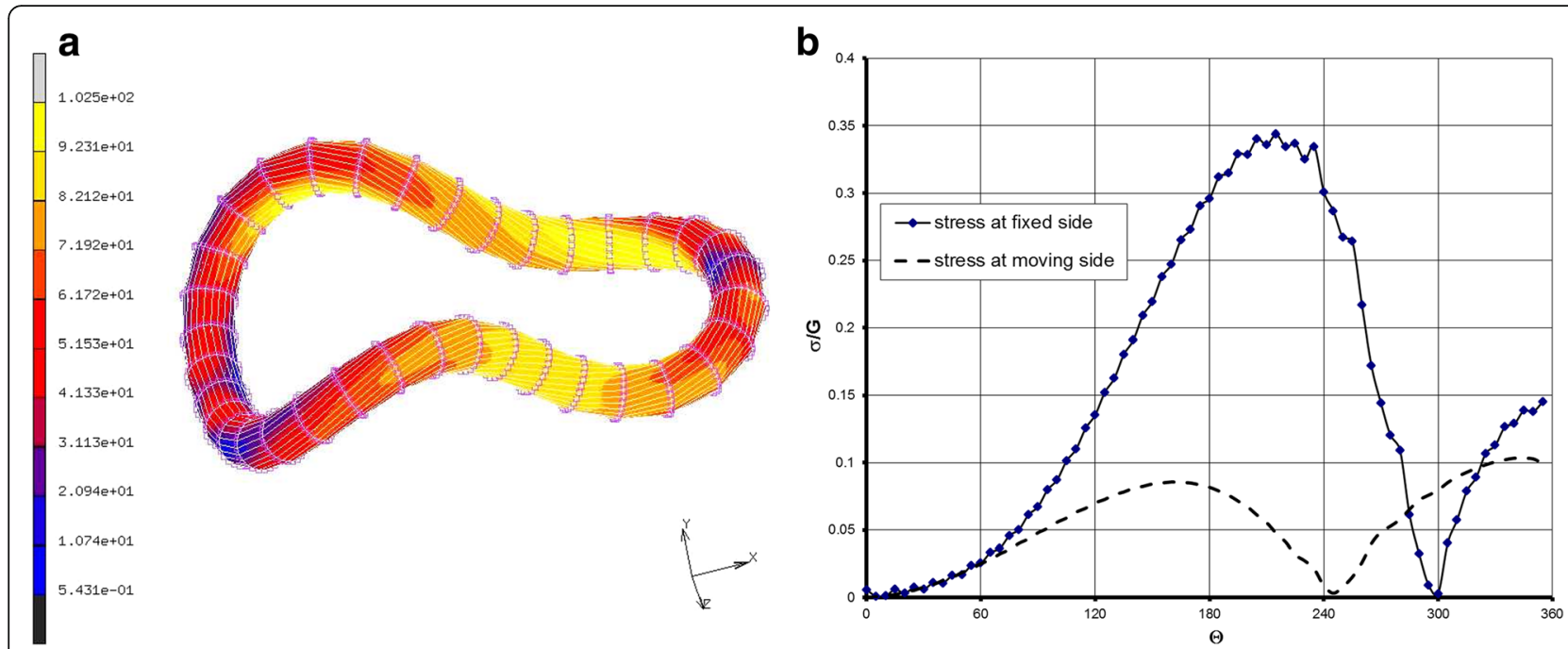

Fig. 7 Hoop stress along major axis for the case of far end twist (a) and stresses on inside and outside circumference of the twisted O-ring (b)

demonstrated that the criterion $\max \left\{\frac{s_{1}}{G}\right\}=\operatorname{Const}(T)$ is the reasonable first order approximation for the life prediction. For room temperature, rough estimates give the safe region $\frac{r_{0}}{R_{0}} \leq 0.25$ making practically any rubber $\mathrm{O}$-ring safe. The situation is changed with an increase in temperature. First of all, the fracture load drops approximately four times if the temperature is $200{ }^{\circ} \mathrm{C}$. Also, due to additional thermal stretch, according to the ArudaBoyce model (see the increase of the slope with stretch ratio in Fig. 3), the shear modulus $G$ increases with temperature. Thus, for a stretch of $20 \%$ the maximum ratio is $\frac{r_{0}}{R_{0}} \approx 0.075$ at $200{ }^{\circ} \mathrm{C}$.

\section{Conclusions}

We have shown that adding a small volume change to classical rubber elastic models can aid in analytical and numerical simulations of polymer structures. Rubber elastic models with volume change, when incorporated in finite element codes, remove the need for special elements. Such models reduce naturally to the case of infinite bulk modulus, and allow a smooth progression from compressible to incompressible materials. Simulations using various rubber elastic models showed that allowing volume changes improves the numerical stability and affects the accuracy of numerical predictions. Model modifications discussed in the paper help to reduce numerical difficulties and readily

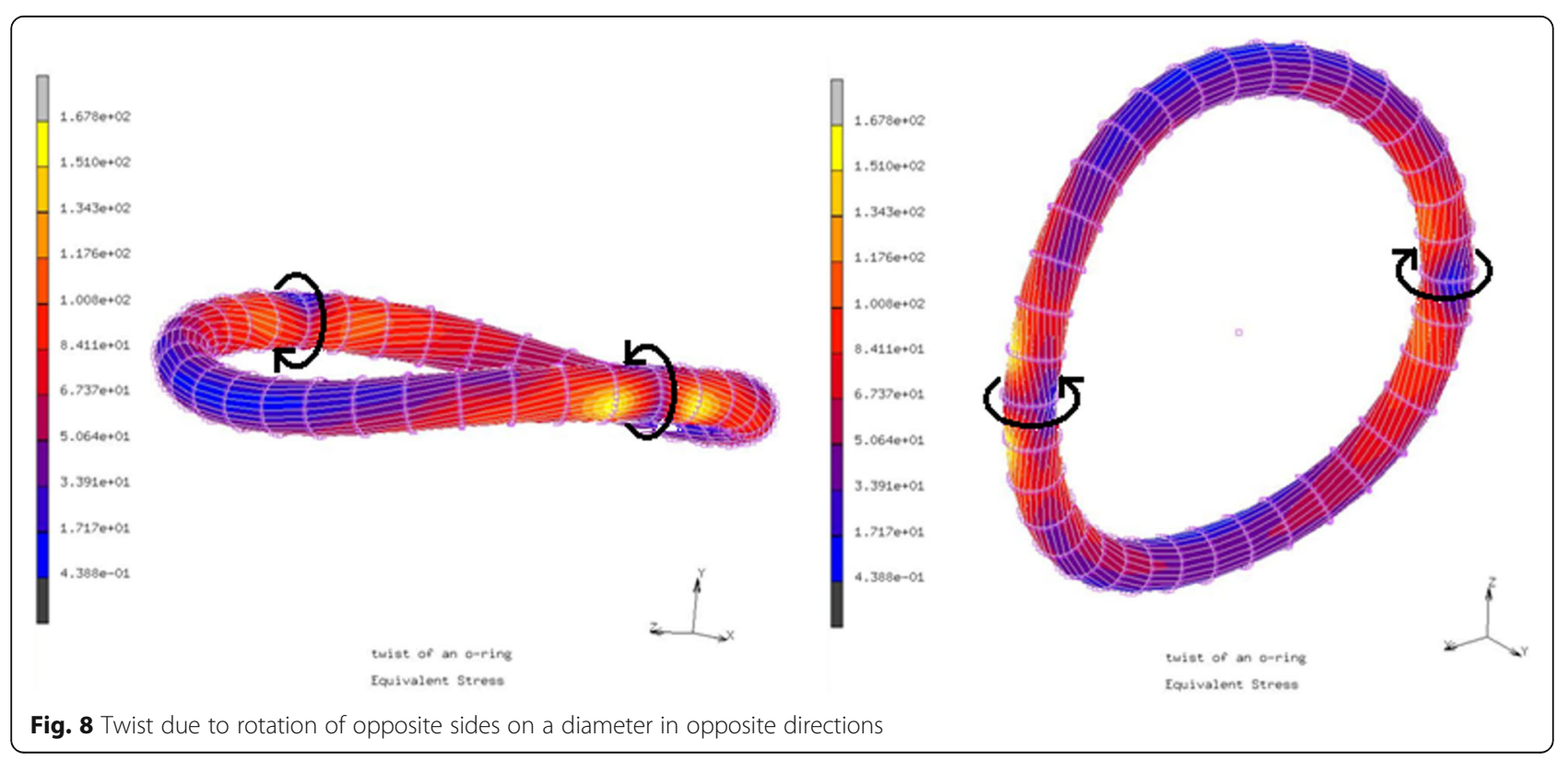




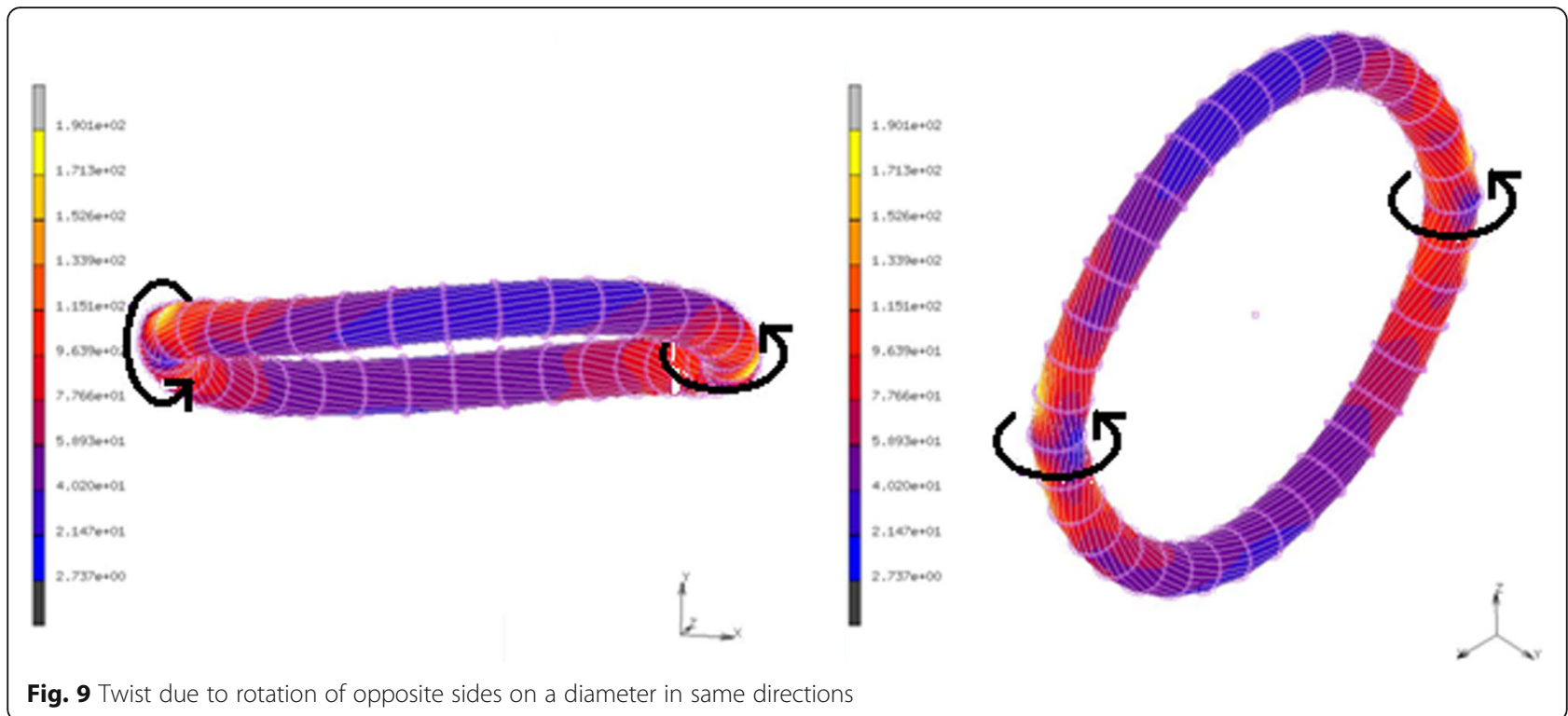

allow the determination of the source of the instabilities. The analysis of stable non-round shapes of twisted O-ring (elastic instability) became numerically stable only after implementing a compressible material model (i.e., the numerical instability is removed).

Simulations using various rubber elastic models showed that allowing volume changes improves the numerical stability. Models that fail to converge leave the analyst with little in the way of information on corrections. We observed that most of the 'failure to converge' results in rubber elastic simulations are due to numerical instabilities. Our model modification helps to reduce numerical difficulties and allows modelers to readily determine the source of the instabilities. We also showed that the modified Aruda-Boyce material model is more accurate than generalized Mooney or Ogden models in stress-strain and stability prediction.

We have evaluated the role of twist in the formation of stress-strain state in O-rings. Such a twist can be related to the installation process when the ring may be placed in a high stress equilibrium state by twisting (and stretching). This causes elastic instabilities resulting in highly deformed O-ring shapes. Numerical results show that pure O-ring twist causes localized hoop stresses up to 0.4G. Numerical analysis using compressible models allowed prediction of the stable deformed states that do not remain round. The O-ring is buckled and reaches a chair-type non-planar shape just beyond inside-out twist. This is an illustration of the fundamental elastic instability. The results indicate that elastic material volume change causes additional stresses mostly associated with an additional hydrostatic pressure. Hence, it is important to use compressible models for accurate stress state predictions. If the ring is set on a rigid cylinder, its shape is defined and additional stresses, sometimes very localized, arise that in turn may lead to the O-ring cracking.

\section{Endnotes}

${ }^{1}$ Other possible application of O-ring stability analysis is elastic models for DNA (Goriely 2006; Benham 1983).

${ }^{2}$ Note that for absolutely incompressible material, this strain energy function is exactly coincident with the incompressible Aruda-Boyce expression. More realistic volume changes would be obtained by replacing $1 / 2 K(J-1)^{2}$ by $1 / 2 K\left[\left(J^{2}-1\right)-\ln (J)\right]$ as shown in Ali et al. (2010).

\section{Appendix}

\section{Conversion of stress and strain components}

The finite element simulations converting from the code strain measure (Green-Lagrange strain) to material model measure (right-hand stretch) and from Biot (Jaumann) stress (See Atluri and Cazzani 1995) of the material model to the second Piola-Kirchoff stress used in the finite element code.

We begin with the deformation gradient

$$
F_{i j}=\frac{\partial x_{i}}{\partial X j}
$$

where $X_{i}$ are the original coordinates and $x_{i}$ are the final coordinates. The difference is the displacement, $u_{i}$, i.e.,

$$
x_{i}=X_{i}+u_{i}
$$

The Green-Lagrange strain, used in the finite element code, is defined by

$$
E_{i j}=\frac{1}{2}\left(F_{k i} F_{k j}-\delta_{i j}\right)
$$

Note that repeated indices are summed. Using Eq. (3) 


$$
E_{i j}=\frac{1}{2}\left(u_{i, j}+u_{j, i}+u_{k, i} u_{k, j}\right) .
$$

The material model uses the right-hand stretch tensor, $U_{i j}$, defined by

$$
F_{i j}=R_{i k} U_{k j}
$$

where $U_{i j}=U_{j i}, R_{i k} R_{j k}=R_{k i} R_{k j}==\delta_{i j}$ and $\delta_{i j}$ is the Kronecker delta. Then

$$
F_{k i} F_{k j}=U_{i k} U_{k j}
$$

where use has been made of the fact that the matrix $U_{i j}$ is symmetric. Substituting Eq. (39) into Eq. (36)

$$
E_{i j}=\frac{1}{2}\left(U_{i k} U_{k j}-\delta_{i j}\right) .
$$

and, hence,

$$
U_{i k} U_{k j}=\delta_{i j}+2 E_{i j}
$$

In matrix form

$$
\stackrel{U^{2}}{\rightarrow}=\underset{I}{\rightarrow}+2 \underline{E}=\underline{A} .
$$

Let diagonal matrix $\underline{\lambda}^{2}$ contain the eigenvalues of $\underline{A}$ and let the corresponding eigenvectors be stored rowwise in $\Phi$, then the right-hand stretch is given by

$\underline{U}=\underline{\Phi} \underline{\lambda} \underline{\Phi}^{T}$. Hence, given the Green-Lagrange strain, we can find the right-hand stretch tensor.

Once the right-hand stretch tensor, and its eigenvalues, is found the material model can be used to find the Biot stress, $S$.

The stress measures must preserve the rate that elastic energy is added will define the stress measures, that is

$$
T_{i j} \dot{E}_{i j}=S_{i j} \dot{U}_{i j}
$$

where $\left(^{\cdot}\right)=d() / d t$ and $T$ is the second Piola-Kirchoff stress tensor. From Eq. (40)

$$
\dot{E}_{i j}=\frac{1}{2}\left(U_{n i} \dot{U}_{n j}+\dot{U}_{n i} U_{n j}\right) .
$$

But the tensor $U_{i j}$ is symmetric making

$$
\dot{E}_{i j}=U_{n i} \dot{U}_{n j} \text {. }
$$

Then Eq. (43) yields

$$
S_{i j}=U_{i k} T_{k j} \text { or } \underline{S}=\underline{U} \underline{T} .
$$

We can now find the second Piola-Kirchkoff stress using

$$
\underline{T}=\underline{U}^{-1} \underline{S} .
$$

\section{Competing interests}

The authors declare that they have no competing interests.

\section{Author details}

${ }^{1}$ UCONN, Department of Mechanical Engineering, 191 Auditorium Rd, U-3139, Storrs, CT 06269-3139, USA. ²United Technologies Research Center, Department of Physical Sciences, 411 Silver Lane, MS129-73, East Hartford, CT 06108, USA.

Received: 22 November 2016 Accepted: 21 January 2017

Published online: 04 February 2017

\section{References}

ADINA (2008) Structures-modeling and theory guide 3.8, pp. 339-341

Ali, A., Housseini, M., \& Sahari, B. B. (2010). A review of constitutive models for rubber-like materials. American Joyrnal of Engineering and Applied Science, 3(1), 232-239.

ANSYS, Inc. (2004) Theory Reference, ANSYS 9.0

Arruda, E. M., \& Boyce, M. C. (1993). A three-dimensional constitutive model for the large stretch behavior of rubber elastic materials. Journal of the Mechanics and Physics of Solids, 41(2), 389-412.

Atluri, S. N., \& Cazzani, A. (1995). Rotations in computational solid mechanics. Archives of Computational Methods in Engineering, 2(1), 49-138.

Benham, C. J. (1983). Geometry and mechanics of DNA superhelicity. Biopolymers, 22, 2477-2495.

Bonet, J., \& Wood, R. D. (1997). Nonlinear mechanics for finite element analysis. Cambridge: Cambridge University Press.

Cassenti, B., \& Staroselsky, A. (2000). Strength and Stability of Rubber O-Ring Seals. In K. Grassie, E. Teuchkholl, G. Wegner, J. Hauser, \& H. Hanselka (Eds.), Functional Materials, 13. Weinheim: Wiley-VCH Verlag GmbH \& Co. KGaA. doi: 10.1002/3527607420.ch56.

Chadwick, P., \& Ogden, R. W. (1971). On the definition of elastic moduli. Archive for Rational Mechanics and Analysis, 44, 41-53.

Destrade, M., Gilchrist, M. D., Motherway, J., \& Murphy, J. G. (2012). Slight compressibility and sensitivity to changes in Poisson's ratio. International Journal for Numerical Methods in Engineering, 90(4), 403-411.

Dolbow, J., \& Belytschko, T. (1999). Volumetric locking in the element free Galerkin method. International Journal for Numerical Methods in Engineering, 46(6), 925-942

Duffet, G., \& Reddy, B. D. (1986). The solution of multi-parameter system of equations with application to problems in nonlinear elasticity. Computer Methods in Applied Mechanics and Engineering, 59, 179-213.

Gent, A. N. (2005). Elastic instabilities in rubber. International Journal of Non-Linear Mechanics, 40(2-3), 165-175.

Gent, A. N., Suh, J. B., \& Kelly, S. G., III. (2007). Mechanics of rubber shear springs. International Journal of Non-Linear Mechanics, 42, 241-249.

Ghaemi, H., Behdinan, K., \& Spence, A. (2006). On the development of compressible pseudo-strain energy density function for elastomers Part 1. Theory and experiment. Journal of Materials Processing Technology, 178, 307-316.

Goriely, A. (2006). Twisted rings and the rediscoveries of Michell's instability. Journal of Elasticity, 84, 281-299.

Gurvich, M., \& Fleischman, T. S. (2003). A simple approach to characterize finite compressibility of elastomers. Rubber Chemistry and Technology, 76, 912-922.

Kamiński, M., \& Lauke, B. (2013). Parameter Sensitivity and Probabilistic Analysis of the Elastic Homogenized Properties for Rubber Filled Polymers. CMES, 93(6), $411-440$.

MARC (2005) MARC reference Library: Volume A User Information, MARC Analysis Research Corporation

Ogden, R. W. (1972). Large deformation isotropic elasticity - on the correlation of theory and experiment for incompressible rubberlike solids. Proceedings of the Royal Society of London A, 326, 565-584.

Pantuso, D., \& Bathe, K.-J. (1997). On the stability of mixed finite elements in large strain analysis of incompressible solids. Finite Elements in Analysis and Design, 28, 83-104.

Reed, K. W., \& Atluri, S. N. (1983) On the generalization of certain rate-type constitutive equations for very large strains. In C. S. Desai, \& R. H. Gallagher (Eds.), Constitutive laws for engineering materials, theory and application (pp. 71-75). College of Engineering, Department of Civil Engineering Mechanics, University of Arizona, Tucson 
Reese, S., \& Wriggers, P. (1995). A finite element method for stability problems in finite elasticity. International Journal for Numerical Methods in Engineering, 38, $1171-1200$

Ritcher, B. (2016). http://www.o-ring-prueflabor.de/en/home/.

Simo, J. C., \& Taylor, R. L. (1991). Quasi-incompressible finite elasticity in principal stretches. Continuum basis and numerical algorithms. Computer Methods in Applied Mechanics and Engineering, 85, 273-310.

Sussman, T., \& Bathe, K.-J. (1987). A finite element formulation for nonlinear incompressible elastic and inelastic analysis. Computers \& Structures, 26, 357-409.

Tabiei, A., \& Khambati, S. (2015). A 3-D visco-hyperelastic constitutive model for rubber with damage for finite element simulation. CMES, 105(1), 25-45.

Treloar, L. R. G. (1975). The Physics of Rubber Elasticity. Oxford: Clarendon Press.

Zéhil, G.P., \& Gavin, H. P. (2013). Unified constitutive modeling of rubber-like materials under diverse loading conditions. International Journal of Engineering Science, 62, 90-105.

\section{Submit your manuscript to a SpringerOpen ${ }^{\circ}$ journal and benefit from:}

- Convenient online submission

- Rigorous peer review

- Immediate publication on acceptance

- Open access: articles freely available online

- High visibility within the field

- Retaining the copyright to your article

Submit your next manuscript at $\boldsymbol{s p r i n g e r o p e n . c o m ~}$ 